\title{
New Ultraspherical Wavelets Spectral Solutions for Fractional Riccati Differential Equations
}

\author{
W. M. Abd-Elhameed ${ }^{1,2}$ and Y. H. Youssri ${ }^{2}$ \\ ${ }^{1}$ Department of Mathematics, Faculty of Science, King Abdulaziz University, Saudi Arabia \\ ${ }^{2}$ Department of Mathematics, Faculty of Science, Cairo University, Giza 12613, Egypt
}

Correspondence should be addressed to W. M. Abd-Elhameed; walee_9@yahoo.com

Received 19 March 2014; Accepted 29 April 2014; Published 22 May 2014

Academic Editor: Ali H. Bhrawy

Copyright @ 2014 W. M. Abd-Elhameed and Y. H. Youssri. This is an open access article distributed under the Creative Commons Attribution License, which permits unrestricted use, distribution, and reproduction in any medium, provided the original work is properly cited.

\begin{abstract}
We introduce two new spectral wavelets algorithms for solving linear and nonlinear fractional-order Riccati differential equation. The suggested algorithms are basically based on employing the ultraspherical wavelets together with the tau and collocation spectral methods. The main idea for obtaining spectral numerical solutions depends on converting the differential equation with its initial condition into a system of linear or nonlinear algebraic equations in the unknown expansion coefficients. For the sake of illustrating the efficiency and the applicability of our algorithms, some numerical examples including comparisons with some algorithms in the literature are presented.
\end{abstract}

\section{Introduction}

The fractional calculus deals with derivatives and integrals to an arbitrary order (real or complex order). Fractional ordinary differential equations are crucial in many fields. For example, many physical phenomena in acoustics, damping laws and electroanalytical chemistry, neuron modeling, diffusion processing, and material sciences (see, e.g., [1-3]) are described by differential equations of fractional order.

The use of fractional differential and integral operators in mathematical models has gained great importance in recent years. Several forms of fractional differential equations have been proposed in standard models, and there has been significant interest in developing numerical schemes for their solutions. There are numerous methods that deal with these types of equations; some of these methods are Laplace transform [4], Adomian decomposition method (ADM) [5], variational iteration method (VIM) [6], fractional difference method (FDM) [7], a quadrature tau method [8], and a shifted Jacobi spectral method [9].

Spectral methods are a class of techniques used extensively in various fields such as applied mathematics and scientific computing (see, e.g., [10-12]). These methods aim to find numerical solutions to different kinds of differential equations. They are used in solving ordinary, partial, and fractional differential equations. When spectral methods are used, the numerical solution is assumed as an expansion in terms of certain special functions which are called "basis functions." A great advantage of spectral methods is that they take on a global approach unlike finite element methods which use a local approach. There are three well-known versions of spectral methods, namely, tau, collocation, and Galerkin methods (see, e.g., [13]). The choice of the suitable spectral method suggested for solving the given equation depends certainly on the type of the differential equation and on the type of the boundary conditions governed by it.

The ultraspherical polynomials have received considerable attention in recent decades, from both theoretical and practical points of view (see, e.g., [14]). Some authors are interested in employing these polynomials for solving various kinds of differential equations. In this respect, Elgindy and Smith-Miles in [15] treated boundary value problems and integral and integrodifferential equations using ultraspherical integration matrices. Moreover, Doha and Abd-Elhameed employed ultraspherical polynomials for solving one- and two-dimensional second-order differential equations [16]. In addition, the same authors in [17] developed some accurate spectral solutions for the parabolic and elliptic partial differential equations based on the ultraspherical tau method. 
The use of wavelets in solving ordinary and fractional differential equations has attracted a large number of authors. For example, in a huge number of articles, Legendre and Chebyshev wavelets are employed for treating ordinary differential equations as well as fractional differential equations (see, e.g., [18-20]). To the best of our knowledge, no articles deal with the application of ultraspherical wavelets in handling fractional-order differential equations. This motivates our interest in such kind of wavelets hoping to employ them in various practical applications. Another motivation is that the Chebyshev and Legendre wavelets can be deduced as special cases of the ultraspherical wavelets.

The fractional-order Riccati differential equation is one of the important fractional-order differential equations. This equation has applications in various disciplines, such as random processes, optimal control, and diffusion problems. We refer the interested readers in the fundamental theories of this equation and its applications to the book of Reid [21].

The main objective in this paper is to introduce two efficient spectral algorithms based on using ultraspherical wavelets for solving fractional-order Riccati differential equation. The two algorithms, namely, ultraspherical wavelets tau method (UWTM) and ultraspherical wavelets collocation method (UWCM), are implemented and presented for handling both of linear and nonlinear fractional-order Riccati differential equations.

The paper is organized as follows. Section 2 is concerned with presenting some necessary definitions and mathematical preliminaries of the fractional calculus theory which are required for establishing our results. Also, in this section, some properties of ultraspherical polynomials and their shifted ones are presented. Section 3 is devoted to constructing the ultraspherical wavelets. In Section 4, some new spectral wavelets solutions for fractional-order Riccati differential equations are developed based on employing the ultraspherical wavelets which were constructed in Section 3. In Section 5, the convergence of the suggested ultraspherical wavelets expansion is carefully investigated. Some numerical examples and discussions are given in Section 6 aiming to illustrate the efficiency, simplicity, and applicability of the suggested algorithms. Some conclusions are given in Section 7.

\section{Preliminaries}

2.1. Basic Definitions of Fractional Calculus. This section is concerned with presenting some notations, definitions, and preliminary facts of the fractional calculus theory which will be useful throughout this paper.

Definition 1. The Riemann-Liouville fractional integral operator $I^{\alpha}$ of order $\alpha$ on the usual Lebesgue space $L^{1}[0,1]$ is defined as

$$
I^{\alpha} f(t)= \begin{cases}\frac{1}{\Gamma(\alpha)} \int_{0}^{t}(t-\tau)^{\alpha-1} f(\tau) d \tau, & \alpha>0 \\ f(t), & \alpha=0 .\end{cases}
$$

The operator $I^{\alpha}$ has the following properties:

$$
\begin{aligned}
& \text { (i) } I^{\alpha} I^{\beta}=I^{\alpha+\beta}, \\
& \text { (ii) } I^{\alpha} I^{\beta}=I^{\beta} I^{\alpha}, \\
& \text { (iii) }\left(I^{\alpha} I^{\beta} f\right)(t)=\left(I^{\beta} I^{\alpha} f\right)(t), \\
& \text { (iv) } I^{\alpha}(t-a)^{\nu}=\frac{\Gamma(\nu+1)}{\Gamma(\nu+\alpha+1)}(t-a)^{\nu+\alpha},
\end{aligned}
$$

where $f \in L^{1}[0,1], \alpha, \beta \geqslant 0$, and $\nu>-1$.

Definition 2. The Riemann-Liouville fractional derivative of order $\alpha>0$ is defined by

$$
\left(D^{\alpha} f\right)(t)=\left(\frac{d}{d t}\right)^{n}\left(I^{n-\alpha} f\right)(t), \quad n-1 \leqslant \alpha<n, n \in \mathbb{N}
$$

The following well-known properties for the operator $D^{\alpha}$ are useful:

$$
\begin{gathered}
D^{\alpha} C=0, \quad C \text { is a constant, } \\
D^{\alpha} t^{\beta}= \begin{cases}0, & \text { for } \beta \in \mathbb{N}_{0}, \beta<\lceil\alpha\rceil, \\
\frac{\Gamma(\beta+1)}{\Gamma(\beta+1-\alpha)} t^{\beta-\alpha}, & \text { for } \beta \in \mathbb{N}_{0}, \beta \geq\lceil\alpha\rceil,\end{cases}
\end{gathered}
$$

where the ceiling notation $\lceil\alpha\rceil$ denotes the smallest integer greater than or equal to $\alpha$ and $\mathbb{N}_{0}=\{0,1,2, \ldots\}$. Recall that, for $\alpha \in \mathbb{N}$, the fractional-differential operator coincides with the usual differential operator of integer-order. Similar to integer-order differentiation, also $D^{\alpha}$ is a linear operator; that is,

$$
D^{\alpha}(\lambda f(t)+\mu g(t))=\lambda D^{\alpha} f(t)+\mu D^{\alpha} g(t),
$$

where $\lambda$ and $\mu$ are constants.

Definition 3. The Caputo definition of fractional differential operator is given by

$$
\begin{array}{r}
\left(D_{*}^{\alpha} f\right)(t)=\frac{1}{\Gamma(n-\alpha)} \int_{0}^{t}(t-\tau)^{n-\alpha-1} f^{(n)}(\tau) d \tau, \\
\alpha>0, \quad t>0,
\end{array}
$$

where $n-1 \leqslant \alpha<n, n \in \mathbb{N}$.

The operator $D_{*}^{\alpha}$ satisfies the following two basic properties for $n-1 \leqslant \alpha<n$ :

$$
\begin{gathered}
\left(D_{*}^{\alpha} I^{\alpha} f\right)(t)=f(t), \\
\left(I^{\alpha} D_{*}^{\alpha} f\right)(t)=f(t)-\sum_{k=0}^{n-1} \frac{f^{(k)}\left(0^{+}\right)}{k !}(t-a)^{k}, \quad t>0 .
\end{gathered}
$$

For more details on mathematical properties of fractional derivatives and integrals, see, for example, [4, 22]. 
2.2. Some Properties of Ultraspherical Polynomials and Their Shifted Ones. The ultraspherical polynomials (a special type of Jacobi polynomials) associated with the real parameter $(\lambda>-1 / 2)$ are a sequence of orthogonal polynomials defined on $(-1,1)$, with respect to the weight function $w(x)=(1-$ $\left.x^{2}\right)^{\lambda-1 / 2}$. The orthogonality relation is given by

$$
\begin{array}{r}
\int_{-1}^{1}\left(1-x^{2}\right)^{\lambda-1 / 2} C_{m}^{(\lambda)}(x) C_{n}^{(\lambda)}(x) d x \\
\quad= \begin{cases}\frac{\sqrt{\pi} n ! \Gamma(\lambda+1 / 2)}{(2 \lambda)_{n}(n+\lambda) \Gamma(\lambda)}, & m=n, \\
0, & m \neq n,\end{cases}
\end{array}
$$

where

$$
(2 \lambda)_{n}=\frac{\Gamma(n+2 \lambda)}{\Gamma(2 \lambda)}
$$

It should be noted here that the ultraspherical polynomials $C_{n}^{(\lambda)}(x)$ are normalized such that $C_{n}^{(\lambda)}(1)=1$. This normalization is characterized by an advantage that the polynomials $C_{n}^{(0)}(x)$ are identical with the Chebyshev polynomials of the first kind $T_{n}(x), C_{n}^{(1 / 2)}(x)$ are the Legendre polynomials $L_{n}(x)$, and $C_{n}^{(1)}(x)$ is equal to $(1 /(n+1)) U_{n}(x)$, where $U_{n}(x)$ are the Chebyshev polynomials of the second kind.

The polynomials $C_{n}^{(\lambda)}(x)$ may be generated by using the recurrence relation

$$
\begin{array}{r}
(n+2 \lambda) C_{n+1}^{(\lambda)}(x)=2(n+\lambda) x C_{n}^{(\lambda)}(x)-n C_{n-1}^{(\lambda)}(x), \\
n=1,2,3, \ldots,
\end{array}
$$

with the initial values $C_{0}^{(\lambda)}(x)=1$ and $C_{1}^{(\lambda)}(x)=x$.

For more properties and relations of ultraspherical polynomials, see, for instance, [23].

The shifted ultraspherical polynomials $\widetilde{C}_{n}^{(\lambda)}(x)=$ $C_{n}^{(\lambda)}(2 x-1)$ are a sequence of orthogonal polynomials defined on $(0,1)$, with respect to the weight function $\widetilde{w}(x)=\left(x-x^{2}\right)^{\lambda-1 / 2}$; that is,

$$
\begin{gathered}
\int_{0}^{1}\left(x-x^{2}\right)^{\lambda-1 / 2} \widetilde{C}_{m}^{(\lambda)}(x) \widetilde{C}_{n}^{(\lambda)}(x) d x \\
\quad= \begin{cases}\frac{\pi 2^{1-4 \lambda} \Gamma(n+2 \lambda)}{n !(n+\lambda)(\Gamma(\lambda))^{2}}, & m=n, \\
0, & m \neq n .\end{cases}
\end{gathered}
$$

They also may be generated by using the recurrence relation

$$
\begin{array}{r}
(n+2 \lambda) \widetilde{C}_{n+1}^{(\lambda)}(x)=2(n+\lambda)(2 x-1) \widetilde{C}_{n}^{(\lambda)}(x)-n \widetilde{C}_{n-1}^{(\lambda)}(x), \\
n=1,2,3, \ldots,
\end{array}
$$

with the initial values $\widetilde{C}_{0}^{(\lambda)}(x)=1$ and $\widetilde{C}_{1}^{(\lambda)}(x)=2 x-1$.

All relations and properties of ultraspherical polynomials can be easily transformed to give the corresponding relations and properties of the shifted ultraspherical polynomials.
Now, the following integral formula (see [23]) is needed:

$$
\int C_{n}^{(\lambda)}(x) w(x) d x=\frac{-2 \lambda\left(1-x^{2}\right)^{\lambda+1 / 2}}{n(n+2 \lambda)} C_{n-1}^{(\lambda+1)}(x), \quad n \geqslant 1 .
$$

Also, the following theorem is essential in investigating the convergence analysis for the suggested ultraspherical wavelets expansion.

Theorem 4 (Bernstein-type inequality; see [24]). The following inequality holds for ultraspherical polynomials:

$$
\begin{aligned}
(\sin \theta)^{\lambda}\left|C_{n}^{(\lambda)}(\cos \theta)\right| & <\frac{2^{1-\lambda} \Gamma(n+3 \lambda / 2)}{\Gamma(\lambda) \Gamma(n+1+\lambda / 2)}, \\
0 & \leqslant \theta \leqslant \pi, \quad 0<\lambda<1 .
\end{aligned}
$$

\section{Construction of Ultraspherical Wavelets}

Wavelets constitute a family of functions constructed from dilation and translation of single function called the mother wavelet. When the dilation parameter $a$ and the translation parameter $b$ vary continuously, we have the following family of continuous wavelets:

$$
\psi_{a, b}(t)=|a|^{-1 / 2} \psi\left(\frac{t-b}{a}\right) \quad a, b \in \mathbb{R}, a \neq 0 .
$$

We define the ultraspherical wavelets $\psi_{n m}^{(\lambda)}(t)=\psi(k, n, m, \lambda, t)$ as they have five arguments: $k, n$ can be assumed to be any positive integer, $m$ is the order for the ultraspherical polynomial, $\lambda$ is the known ultraspherical parameter, and $t$ is the normalized time. Explicitly, they are defined on the interval $[0,1]$ as

$\psi_{n m}^{(\lambda)}(t)= \begin{cases}2^{k / 2} \xi_{m} C_{m}^{(\lambda)}\left(2^{k-1} t-n+1\right), & t \in\left[\frac{n-1}{2^{k-1}}, \frac{n}{2^{k-1}}\right], \\ 0, & \text { otherwise, }\end{cases}$

where $m=0,1, \ldots, M-1, n=1,2, \ldots, 2^{k-1}$, and

$$
\xi_{m}=2^{\lambda} \Gamma(\lambda) \sqrt{\frac{m !(m+\lambda)}{2 \pi \Gamma(m+2 \lambda)}} .
$$

Remark 5. It is worth noting here that $\psi_{n m}^{(1 / 2)}(t)$ is identical to Legendre wavelets $[25,26], \psi_{n m}^{(0)}(t)$ is identical to first kind Chebyshev wavelets $[27,28]$, and $\psi_{n m}^{(1)}(t)$ is identical to second kind Chebyshev wavelets $[29,30]$.

Now, consider a function $f(t)$ defined on $[0,1]$ and suppose that $f(t)$ may be expanded in terms of the ultraspherical wavelets as

$$
f(t)=\sum_{n=1}^{\infty} \sum_{m=0}^{\infty} c_{n m} \psi_{n m}^{(\lambda)}(t)
$$


where

$$
c_{n m}=\left(f(t), \psi_{n m}^{(\lambda)}(t)\right)_{\widetilde{w}}=\int_{0}^{1}\left(t-t^{2}\right)^{\lambda-1 / 2} f(t) \psi_{n m}^{(\lambda)}(t) d t .
$$

Also, we can assume that the function $f(t)$ can be approximated in terms of ultraspherical wavelets as

$$
f(t) \approx \sum_{n=1}^{2^{k-1}} \sum_{m=0}^{M-1} c_{n m} \psi_{n m}^{(\lambda)}(t)
$$

\section{Spectral Solutions of Fractional-Order Riccati Differential Equation}

In this section, with the aid of the ultraspherical wavelets expansion together with the two well-known spectral methods, namely, tau and collocation methods, we numerically solve the following fractional-order Riccati differential equation:

$$
\begin{array}{r}
D^{\alpha} u(t)=p(t) u(t)^{2}+q(t) u(t)+r(t), \\
t \in(0,1), \quad 0<\alpha \leqslant 1,
\end{array}
$$

subject to the initial condition

$$
u(0)=B .
$$

The following lemma is needed for developing the wavelets solutions of fractional-order Riccati differential equation.

Lemma 6. The fractional derivative of order $\alpha \in(\nu-1, \nu)$ of the ultraspherical wavelets (16) is given by

$$
D^{\alpha} \psi_{n m}^{(\lambda)}(t)=\left\{\begin{array}{l}
\frac{2^{1+(\alpha-\nu+1 / 2) k} \xi_{m}}{\Gamma(\alpha-\nu)}\left(\frac{d}{d t}\right)^{\nu} \\
\quad \times \int_{0}^{2^{k-1} t-n+1} \frac{\widetilde{C}_{m}^{(\lambda)}(\tau)}{\left(2^{k-1} t-n+1-\tau\right)^{\alpha-\nu+1}} d \tau, \\
\quad t \in\left[\frac{n-1}{2^{k-1}}, \frac{n}{2^{k-1}}\right], \\
0, \quad \text { otherwise. }
\end{array}\right.
$$

Proof. The result can be immediately obtained by applying the operator defined in (3) on the ultraspherical wavelets $\psi_{n m}^{(\lambda)}(t)$ defined in (16).

4.1. Case I: Linear Fractional-Order Riccati Differential Equation. In this subsection, we consider (21) in case of $p(t)=0$; that is, our aim is to discuss the following fractional-order Riccati linear differential equation:

$$
D^{\alpha} u(t)=q(t) u(t)+r(t), \quad t \in(0,1), 0<\alpha \leqslant 1,
$$

subject to the initial condition (22).

Now, assume that the approximate solution of (24) can be expanded in terms of the ultraspherical wavelets as

$$
u_{k, M, \lambda}(t)=\sum_{n=1}^{2^{k-1}} \sum_{m=0}^{M-1} c_{n m} \psi_{n m}^{(\lambda)}(t)
$$

and if we substitute (25) into (24), then the residual of this equation is given by

$$
R(t)=\sum_{n=1}^{2^{k-1}} \sum_{m=0}^{M-1} c_{n m}\left(D^{\alpha} \psi_{n m}^{(\lambda)}(t)-q(t) \psi_{n m}^{(\lambda)}(t)\right)-r(t) .
$$

The application of the typical tau method (see, e.g., [13]) implies that

$$
\int_{0}^{1} R(t) \psi_{n m}^{(\lambda)}(t) d t=0, \quad(n, m) \in \Lambda,
$$

where

$$
\Lambda=\left\{1,2, \ldots, 2^{k-1}\right\} \times\{0,1, \ldots, M-1\}-\left\{\left(2^{k-1}, M-1\right)\right\} .
$$

Moreover, the use of the initial condition (22) yields

$$
\sum_{n=1}^{2^{k-1} M-1} \sum_{m=0} c_{n m} \psi_{n m}^{(\lambda)}(0)=B
$$

Equations (27) and (29) generate a set consisting of $\left(2^{k-1} M\right)$ equations in the unknown expansion coefficients, $\left\{c_{n m}: 1 \leq\right.$ $\left.n \leq 2^{k-1} ; 0 \leq m \leq M-1\right\}$. This system of equations can be solved with the aid of any suitable solver such as GaussSeidel method (see, [31]). Hence, the unknown components of the vector $C$ can be obtained, and accordingly the required spectral wavelets solution $u_{k, M, \lambda}(t)$ given by $(25)$ can be obtained.

\subsection{Case II: Nonlinear Fractional-Order Riccati Differential} Equation. In this subsection, we aim to handle the following nonlinear fractional-order Riccati differential equation:

$$
\begin{array}{r}
D^{\alpha} u(t)=p(t) u(t)^{2}+q(t) u(t)+r(t), \\
t \in(0,1), \quad 0<\alpha \leqslant 1, \quad p(t) \neq 0,
\end{array}
$$

subject to the initial condition (22).

Assume that the approximate solution of (30) can be expanded in terms of the ultraspherical wavelets as in (25). Substitution of (25) into (30) enables one to write the residual of (30) as

$$
\begin{gathered}
R(t)=\sum_{n=0}^{2^{k-1}} \sum_{m=0}^{M-1} c_{n m} D^{\alpha} \psi_{n m}^{(\lambda)}(t)-p(t)\left(\sum_{n=0}^{2^{k-1} M-1} \sum_{m=0} c_{n m} \psi_{n m}^{(\lambda)}(t)\right)^{2} \\
-q(t)\left(\sum_{n=0}^{2^{k-1} M-1} \sum_{m=0}^{M} c_{n m} \psi_{n m}^{(\lambda)}(t)\right)-r(t) .
\end{gathered}
$$

The application of the collocation method (see, e.g., [13]) implies that

$$
R\left(t_{i}\right)=0, \quad i=1,2, \ldots, 2^{k-1} M-1,
$$


where the points $t_{i}, i=1,2, \ldots, 2^{k-1} M-1$ are selected to be the first $\left(2^{k-1} M-1\right)$ roots of $C_{2^{k-1} M}^{(\lambda)}(t)$. Equations (29) and (32) generate a set of $\left(2^{k-1} M\right)$ equations in the unknown expansion coefficients $\left\{c_{n m}: 1 \leq n \leq 2^{k-1} ; 0 \leq m \leq\right.$ $M-1\}$. This system of equations can be solved with the aid of Newton's iterative method (see [32]), to obtain the unknown components of the vector $C$, and hence the required spectral wavelets solution $u_{k, M, \lambda}(t)$ given by (25) can be obtained.

\section{Convergence Analysis}

In this section, we state and prove the following important theorem which ascertains that the ultraspherical wavelets expansion of a function $f(x)$ with bounded second derivative converges uniformly to $f(x)$.

Theorem 7. A function $f(t) \in L_{\widetilde{\omega}}^{2}[0,1], \widetilde{\omega}=\left(t-t^{2}\right)^{\lambda-1 / 2}$, $0<\lambda<1$, can be expanded as an infinite series of ultraspherical wavelets, which converges uniformly to $f(x)$, provided $\left|f^{\prime \prime}(t)\right| \leqslant L$. Explicitly, the expansion coefficients in (20) satisfy the inequality

$$
\left|c_{n m}\right|<\frac{4 L(1+\lambda)^{2}(m+1+\lambda)^{2}}{(m-2)^{4} n^{5 / 2}}, \quad \forall n \geqslant 1, m>2 .
$$

Proof. From relations (16) and (19), one can write

$$
\begin{aligned}
c_{n m}= & 2^{k / 2} \xi_{m, \lambda} \\
& \times \int_{(n-1) / 2^{k-1}}^{n / 2^{k-1}} f(t) C_{m}^{(\lambda)}\left(2^{k} t-2 n-1\right) \omega\left(2^{k} t-n\right) d t,
\end{aligned}
$$

and the integration of the right hand side of (34) by parts with the aid of relation (13) yields

$$
\begin{aligned}
c_{n m}= & \frac{2^{(6-k) / 2} \lambda \xi_{m, \lambda}}{m(m+2 \lambda)} \\
& \quad \times \int_{(n-1) / 2^{k-1}}^{n / 2^{k-1}} f^{\prime}(t) C_{m-1}^{(\lambda+1)}\left(2^{k} t-2 n-1\right)\left(2^{k} t-n\right) \\
& \quad \times\left(1-2^{k} t+n\right) \omega\left(2^{k} t-n\right) d t .
\end{aligned}
$$

If we integrate (35) by parts again, and make use of the substitution: $2^{k} t-2 n-1=\cos \theta$, then the coefficient $c_{n m}$ takes the form

$$
\begin{aligned}
& c_{n m} \\
& =\frac{\sqrt{2}(\lambda)_{2} \xi_{m, \lambda}}{2^{2 \lambda+(5 k-5) / 2}(m-1)_{2}(m+2 \lambda-1)_{2}} \\
& \quad \times \int_{0}^{\pi} f^{\prime \prime}\left(\frac{1+2 n+\cos \theta}{2^{k}}\right) C_{m-2}^{(\lambda+2)}(\cos \theta)(\sin \theta)^{2 \lambda+4} d \theta .
\end{aligned}
$$

Now, assuming that $m>2$, taking into account the assumption $\left|f^{\prime \prime}(t)\right| \leqslant L$, and with the aid of Theorem 4 , we obtain

$$
\begin{aligned}
& \left|c_{n m}\right| \\
& \leqslant \frac{\sqrt{2}\left|(\lambda)_{2} \xi_{m, \lambda}\right|}{2^{2 \lambda+(5 k-5) / 2}(m-1)_{2}(m+2 \lambda-1)_{2}} \\
& \times \int_{0}^{\pi}\left|f^{\prime \prime}\left(\frac{1+2 n+\cos \theta}{2^{k}}\right)\right|\left|C_{m-2}^{(\lambda+2)}(\cos \theta)\right|(\sin \theta)^{2 \lambda+4} d \theta \\
& \leqslant \frac{\sqrt{2} L|\lambda|(1+\lambda)\left|\xi_{m, \lambda}\right|}{2^{2 \lambda+(5 k-5) / 2}(m-1)_{2}(m+2 \lambda-1)_{2}} \\
& \times \int_{0}^{\pi}\left|C_{m-2}^{(\lambda+2)}(\cos \theta)\right|(\sin \theta)^{2 \lambda+4} d \theta \\
& <\left(\sqrt{2 \pi} L|\lambda|(1+\lambda)\left|\xi_{m, \lambda}\right| \Gamma\left(m+1+\frac{3 \lambda}{2}\right) \Gamma\left(\frac{3+\lambda}{2}\right)\right) \\
& \times\left(2^{3 \lambda+(5 k-5) / 2}(m-1)_{2}(m+2 \lambda-1)_{2}\right. \\
& \left.\times \Gamma(\lambda+2) \Gamma\left(m+\frac{\lambda}{2}\right) \Gamma\left(2+\frac{\lambda}{2}\right)\right)^{-1} .
\end{aligned}
$$

Knowing that $\lambda>0, n<2^{k-1}$, and with the aid of relation (17), we get

$\left|c_{n m}\right|$

$<\frac{2 L|\lambda|(1+\lambda) \Gamma((3+\lambda) / 2) \Gamma(m+1+3 \lambda / 2) \sqrt{m !(m+\lambda)}}{4^{\lambda} \Gamma(2+\lambda / 2) \Gamma(m+\lambda / 2) \sqrt{\Gamma(m+2 \lambda)}(m-2)^{4} n^{5 / 2}}$

$<\frac{4 L(1+\lambda)^{2} \Gamma(m+1+3 \lambda / 2) \sqrt{m !(m+\lambda)}}{\Gamma(m+\lambda / 2) \sqrt{\Gamma(m+2 \lambda)}(m-2)^{4} n^{5 / 2}}$

$<\frac{4 L(1+\lambda)^{2}(m+1+\lambda)^{2}}{(m-2)^{4} n^{5 / 2}}$.

This completes the proof of the theorem.

Note. It is to be noted here that, for large values of $m$ and $n$, and making use of the well-known Stirling's formula (see, [33]), it can be easily shown that $\left|c_{n m}\right|$ is of $\mathcal{O}\left(n^{-5 / 2} m^{-2}\right)$.

\section{Numerical Results and Comparisons}

In this section, we apply the presented algorithms in Section 4, for obtaining numerical solutions for linear and nonlinear fractional-order Riccati differential equations. In addition, we compare our spectral ultraspherical algorithms with some other numerical algorithms that exist in the literature aiming to demonstrate the efficiency and rapid convergence of the proposed algorithms. 
TABLE 1: Comparison between UWCM, GEM, and LAPM for Example 8 for $\alpha=1$.

\begin{tabular}{lcccccc}
\hline$t$ & GEM & LAPM & UWCM & Exact solution & LAPM error & UWCM error \\
\hline 0.1 & 0.1000000000 & 0.1102952044 & 0.1102951969 & 0.1102951969 & $7.5 \cdot 10^{-9}$ & $1.4 \cdot 10^{-12}$ \\
0.2 & 0.2419000000 & 0.2419783394 & 0.2419767996 & 0.2419767996 & $1.5 \cdot 10^{-6}$ & $2.7 \cdot 10^{-12}$ \\
0.3 & 0.3580039000 & 0.3951442714 & 0.3951048487 & 0.3951048487 & $3.9 \cdot 10^{-5}$ & $4.9 \cdot 10^{-12}$ \\
0.4 & 0.5167880007 & 0.5682377001 & 0.5678121663 & 0.5678121663 & $4.2 \cdot 10^{-4}$ & $6.8 \cdot 10^{-12}$ \\
0.5 & 0.6934386 & 0.7588607194 & 0.7580143934 & 0.7580143934 & $8.4 \cdot 10^{-4}$ & $9.1 \cdot 10^{-12}$ \\
\hline
\end{tabular}

TABLE 2: Comparison between UWCM, HPM, and VIM for Example 8 for $\alpha=1 / 2,3 / 4$.

\begin{tabular}{|c|c|c|c|c|c|c|}
\hline \multirow{2}{*}{$t$} & \multicolumn{3}{|c|}{$\alpha=1 / 2$} & \multicolumn{3}{|c|}{$\alpha=3 / 4$} \\
\hline & HPM & VIM & UWCM & HPM & VIM & UWCM \\
\hline 0.1 & 0.321730 & 0.592833 & 0.580926 & 0.216866 & 0.245446 & 0.244563 \\
\hline 0.3 & 0.940941 & 1.174069 & 1.12057 & 0.654614 & 0.710050 & 0.710318 \\
\hline 0.5 & 1.549439 & 1.473790 & 1.45668 & 1.132763 & 1.149016 & 1.15155 \\
\hline 0.7 & 2.066523 & 1.646302 & 1.63391 & 1.594278 & 1.491949 & 1.49335 \\
\hline 0.9 & 2.396839 & 1.756349 & 1.75008 & 1.962239 & 1.730575 & 1.73018 \\
\hline
\end{tabular}

Example 8. Consider the nonlinear Riccati differential equation (see, [34-36])

$$
\begin{gathered}
D^{\alpha} u(t)=1+2 u(t)-u(t)^{2} \\
t \in(0,1], \quad u(0)=0, \quad 0<\alpha \leq 1 .
\end{gathered}
$$

The exact solution for (39), when $\alpha=1$, is given by

$$
u(t)=\sqrt{2} \tanh \left(\sqrt{2} t-\tanh ^{-1}\left(\frac{1}{\sqrt{2}}\right)\right)+1 .
$$

In Table 1, we compare the numerical solution resulting from the application of UWCM, for the case that corresponds to $\lambda=k=1, M=10$, with the generalized Euler method (GEM) and Laplace-Adomian-Padé method (LAPM) [34], while in Table 2, we compare the solution resulting from the application of UWCM, for the case that corresponds to $\lambda=$ $0, k=2, M=4$, with the two solutions resulting from the application of homotopy perturbation method (HPM) developed in [35] and the variational iteration method (VIM) developed in [36]. In addition, Figure 1 illustrates different solutions for Example 8 in case of $k=\lambda=\alpha=1$ for various values of $M$.

Example 9. Consider the nonlinear Riccati differential equation (see, $[36,37])$

$$
\begin{gathered}
D^{\alpha} u(t)=1-u(t)^{2}, \\
t \in(0,1], \quad u(0)=0, \quad 0<\alpha \leq 1 .
\end{gathered}
$$

The exact solution of (41), when $\alpha=1$, is $u(t)=\tanh t$. In Table 3, the maximum absolute error $E$ is listed for the case that corresponds to $\alpha=k=1$, and for various values of $M$, while in Table 4 , we give a comparison between the numerical solution resulting from the application of UWCM, for the case that corresponds to $\lambda=1 / 2, k=2$, $M=5$, with the two numerical solutions resulting from the

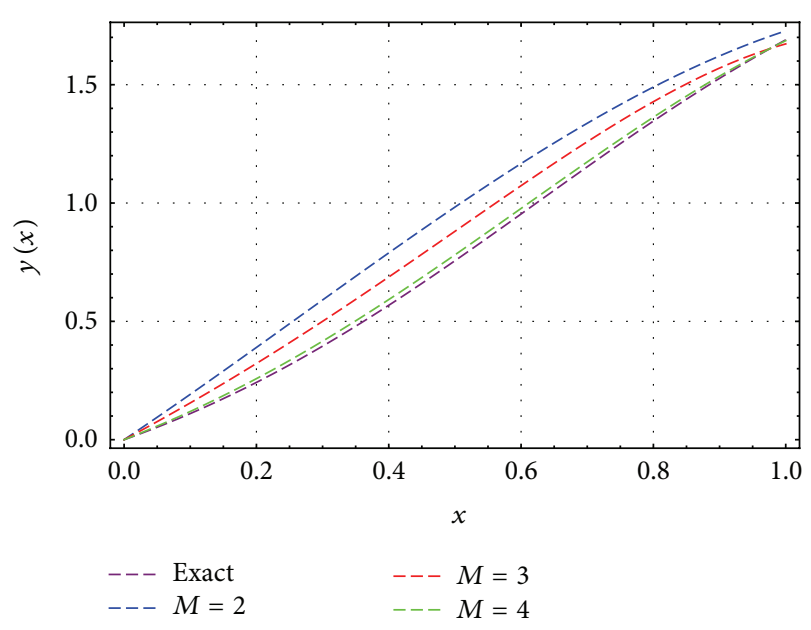

FIGURE 1: Different solutions of Example 8.

application of the two methods, namely, variational iteration method (VIM) and second kind Chebyshev wavelets method (CUWM) developed, respectively, in [36, 37].

Remark 10. It is worth noting here that the results displayed in Table 3 show that the best absolute error resulting from the application of UWCM does not exceed $\left(3 \times 10^{-16}\right)$, while the best error resulting from the application of the two methods, namely, VIM and CUWM developed in [36, 37], does not exceed $\left(1.5 \times 10^{-7}\right)$. Moreover, the results presented in Table 4 appear to agree closely where the UWCM deviates somewhat, because of the expected high accuracy of the UWCM if compared with the results obtained by VIM and CUWM $[36,37]$.

Remark 11. The results of Table 3 ascertain that the results that correspond to the first kind of Chebyshev wavelets expansion (in case of $\lambda=0$ ) are not always better than the other ultraspherical wavelets expansions (see, $[17,38]$ ). 
TABLE 3: Maximum absolute error $E$ for Example 9.

\begin{tabular}{lccccccccc}
\hline$\lambda$ & $M$ & $E$ & $M$ & $E$ & $M$ & $E$ & $M$ & $E$ & $M$ \\
\hline 1 & & $2.76 \cdot 10^{-6}$ & & $3.72 \cdot 10^{-8}$ & & $5.17 \cdot 10^{-11}$ & & $1.44 \cdot 10^{-13}$ & \\
$1 / 2$ & 5 & $3.77 \cdot 10^{-6}$ & & $5.09 \cdot 10^{-9}$ & & $1.32 \cdot 10^{-11}$ & & $3.26 \cdot 10^{-14}$ & 17 \\
0 & $3.03 \cdot 10^{-6}$ & 8 & $1.28 \cdot 10^{-8}$ & 11 & $2.90 \cdot 10^{-11}$ & 14 & $7.24 \cdot 10^{-14}$ & $2.22 \cdot 10^{-16}$ \\
-0.49 & & $5.34 \cdot 10^{-6}$ & & $1.87 \cdot 10^{-8}$ & & $5.72 \cdot 10^{-11}$ & & $3.80 \cdot 10^{-14}$ & $2.44 \cdot 10^{-16}$ \\
\hline
\end{tabular}

TABLE 4: Comparison between UWCM, VIM, and CUWM for Example 9 for $\alpha=1 / 2,3 / 4$.

\begin{tabular}{lcccccc}
\hline$t$ & & $\alpha=1 / 2$ & & & $\alpha=3 / 4$ \\
& VIM & CUWM & UWCM & VIM & CUWM & UWCM \\
\hline 0.2 & 0.436839 & 0.436737 & 0.430766 & 0.309976 & 0.309886 & 0.310516 \\
0.4 & 0.553782 & 0.553802 & 0.556613 & 0.481632 & 0.481638 \\
0.6 & 0.621014 & 0.621026 & 0.645945 & 0.597783 & 0.597790 & 0.5996696 \\
0.8 & 0.666020 & 0.666016 & 0.666657 & 0.678850 & 0.678835 & 0.678914 \\
\hline
\end{tabular}

Example 12. Consider the composite fractional oscillation equation (see, [36])

$$
D^{1 / 4} u(t)+u(t)=t^{2}+\frac{2}{\Gamma(11 / 4)} t^{7 / 4}, \quad u(0)=0
$$

The exact solution for (42) is $u(t)=t^{2}$. We apply the UWTM for problem (42), for the case that corresponds to $k=1, M=$ 3 . In detail, the approximate solution $u_{1,3, \lambda}(t)$ is given by

$$
\begin{aligned}
u_{1,3, \lambda}(t) & =c_{1,0} \psi_{1,0}^{(\lambda)}(t)+c_{1,1} \psi_{1,1}^{(\lambda)}(t)+c_{1,2} \psi_{1,2}^{(\lambda)}(t) \\
& =c_{0} C_{0}^{(\lambda)}(t)+c_{1} C_{1}^{(\lambda)}(t)+c_{2} C_{2}^{(\lambda)}(t) \\
& =c_{0}+c_{1}(2 t-1)+c_{2}\left(\frac{1+2 \lambda-8(1+\lambda)\left(t-t^{2}\right)}{1+2 \lambda}\right),
\end{aligned}
$$

where, $c_{i}=c_{1, i} \xi_{i}, \xi_{i}$ is as given in (17), $i=0,1,2$.

In virtue of (27), the solution of the resulting linear system is

$$
c_{0}=\frac{3+2 \lambda}{8+8 \lambda}, \quad c_{1}=\frac{1}{2}, \quad c_{2}=\frac{1+2 \lambda}{8+8 \lambda},
$$

and consequently

$$
\begin{aligned}
u(t)= & \frac{3+2 \lambda}{8+8 \lambda}+\frac{1}{2}(2 t-1) \\
& +\frac{1+2 \lambda}{8+8 \lambda}\left(\frac{1+2 \lambda-8(1+\lambda)\left(t-t^{2}\right)}{1+2 \lambda}\right)=t^{2}
\end{aligned}
$$

which is the exact solution.

\section{Conclusions}

This paper is concerned with presenting and implementing new algorithms for obtaining numerical spectral solutions for fractional-order Riccati differential equations. These methods are essentially based on employing the ultraspherical wavelets along with the application of the two spectral methods, namely, tau and collocation methods. The developed algorithms are applicable and easy in implementation and of high efficiency. The obtained numerical results indicate that the first kind of Chebyshev wavelets expansion is not always better than the other ultraspherical wavelets expansions.

\section{Conflict of Interests}

The authors declare that there is no conflict of interests regarding the publication of this paper.

\section{Acknowledgment}

This work was funded by the Deanship of Scientific Research (DSR), King Abdulaziz University, Jeddah, under Grant no. (130-269-D1435). The authors, therefore, acknowledge with thanks DSR technical and financial support.

\section{References}

[1] Q. M. Al-Mdallal, M. I. Syam, and M. N. Anwar, "A collocationshooting method for solving fractional boundary value problems," Communications in Nonlinear Science and Numerical Simulation, vol. 15, no. 12, pp. 3814-3822, 2010.

[2] Y. Çenesiz, Y. Keskin, and A. Kurnaz, "The solution of the Bagley-Torvik equation with the generalized Taylor collocation method," Journal of the Franklin Institute: Engineering and Applied Mathematics, vol. 347, no. 2, pp. 452-466, 2010.

[3] V. Daftardar-Gejji and H. Jafari, "Adomian decomposition: a tool for solving a system of fractional differential equations," Journal of Mathematical Analysis and Applications, vol. 301, no. 2, pp. 508-518, 2005.

[4] I. Podlubny, Fractional Differential Equations: An Introduction to Fractional Derivatives, Fractional Differential Equations, to Methods of their Solution and some of their Applications, vol. 198 of Mathematics in Science and Engineering, Academic Press, New York, NY, USA, 1999.

[5] H. Jafari and S. Seifi, "Solving a system of nonlinear fractional partial differential equations using homotopy analysis method," 
Communications in Nonlinear Science and Numerical Simulation, vol. 14, no. 5, pp. 1962-1969, 2009.

[6] S. Das, "Analytical solution of a fractional diffusion equation by variational iteration method," Computers \& Mathematics with Applications, vol. 57, no. 3, pp. 483-487, 2009.

[7] M. M. Meerschaert and C. Tadjeran, "Finite difference approximations for two-sided space-fractional partial differential equations," Applied Numerical Mathematics: An IMACS Journal, vol. 56, no. 1, pp. 80-90, 2006.

[8] A. H. Bhrawy, A. S. Alofi, and S. S. Ezz-Eldien, "A quadrature tau method for fractional differential equations with variable coefficients," Applied Mathematics Letters, vol. 24, no. 12, pp. 2146-2152, 2011.

[9] E. H. Doha, A. H. Bhrawy, D. Baleanu, and S. S. Ezz-Eldien, "On shifted Jacobi spectral approximations for solving fractional differential equations," Applied Mathematics and Computation, vol. 219, no. 15, pp. 8042-8056, 2013.

[10] W. M. Abd-Elhameed, E. H. Doha, and Y. H. Youssri, "Efficient spectral-Petrov-Galerkin methods for third- and fifth-order differential equations using general parameters generalized Jacobi polynomials," Quaestiones Mathematicae, vol. 36, no. 1, pp. 15-38, 2013.

[11] E. H. Doha, W. M. Abd-Elhameed, and Y. H. Youssri, "Second kind Chebyshev operational matrix algorithm for solving differential equations of Lane-Emden type," New Astronomy, vol. 23-24, pp. 113-117, 2013.

[12] E. H. Doha, W. M. Abd-Elhameed, and M. A. Bassuony, "New algorithms for solving high even-order differential equations using third and fourth Chebyshev-Galerkin methods," Journal of Computational Physics, vol. 236, pp. 563-579, 2013.

[13] C. Canuto, M. Y. Hussaini, A. Quarteroni, and T. A. Zang, Spectral Methods in Fluid Dynamics, Springer Series in Computational Physics, Springer, New York, NY, USA, 1988.

[14] K. T. Elgindy and K. A. Smith-Miles, "On the optimization of Gegenbauer operational matrix of integration," Advances in Computational Mathematics, vol. 39, no. 3-4, pp. 511-524, 2013.

[15] K. T. Elgindy and K. A. Smith-Miles, "Solving boundary value problems, integral, and integro-differential equations using Gegenbauer integration matrices," Journal of Computational and Applied Mathematics, vol. 237, no. 1, pp. 307-325, 2013.

[16] E. H. Doha and W. M. Abd-Elhameed, "Efficient spectralGalerkin algorithms for direct solution of second-order equations using ultraspherical polynomials," SIAM Journal on Scientific Computing, vol. 24, no. 2, pp. 548-571, 2002.

[17] E. H. Doha and W. M. Abd-Elhameed, "Accurate spectral solutions for the parabolic and elliptic partial differential equations by the ultraspherical tau method," Journal of Computational and Applied Mathematics, vol. 181, no. 1, pp. 24-45, 2005.

[18] W. M. Abd-Elhameed, E. H. Doha, and Y. H. Youssri, "New wavelets collocation method for solving second-order multipoint boundary value problems using Chebyshev polynomials of third and fourth kinds," Abstract and Applied Analysis, vol. 2013, Article ID 542839, 9 pages, 2013.

[19] L. Zhu and Q. Fan, "Solving fractional nonlinear Fredholm integro-differential equations by the second kind Chebyshev wavelet," Communications in Nonlinear Science and Numerical Simulation, vol. 17, no. 6, pp. 2333-2341, 2012.

[20] I. Sadek, T. Abualrub, and M. Abukhaled, "A computational method for solving optimal control of a system of parallel beams using Legendre wavelets," Mathematical and Computer Modelling, vol. 45, no. 9-10, pp. 1253-1264, 2007.
[21] W. T. Reid, Riccati Differential Equations, vol. 86 of Mathematics in Science and Engineering, Academic Press, New York, NY, USA, 1972.

[22] K. B. Oldham and J. Spanier, The Fractional Calculus, Academic Press, New York, NY, USA, 1974.

[23] G. E. Andrews, R. Askey, and R. Roy, Special Functions, vol. 71 of Encyclopedia of Mathematics and its Applications, Cambridge University Press, Cambridge, UK, 1999.

[24] C. Giordano and A. Laforgia, "On the Bernstein-type inequalities for ultraspherical polynomials," Journal of Computational and Applied Mathematics, vol. 153, pp. 243-248, 2003.

[25] M. Razzaghi and S. Yousefi, "Legendre wavelets direct method for variational problems," Mathematics and Computers in Simulation, vol. 53, no. 3, pp. 185-192, 2000.

[26] S. A. Yousefi, "Legendre wavelets method for solving differential equations of Lane-Emden type," Applied Mathematics and Computation, vol. 181, no. 2, pp. 1417-1422, 2006.

[27] E. Babolian and F. Fattahzadeh, "Numerical solution of differential equations by using Chebyshev wavelet operational matrix of integration," Applied Mathematics and Computation, vol. 188, no. 1, pp. 417-426, 2007.

[28] Y. Li, "Solving a nonlinear fractional differential equation using Chebyshev wavelets," Communications in Nonlinear Science and Numerical Simulation, vol. 15, no. 9, pp. 2284-2292, 2010.

[29] K. Maleknejad, S. Sohrabi, and Y. Rostami, "Numerical solution of nonlinear Volterra integral equations of the second kind by using Chebyshev polynomials," Applied Mathematics and Computation, vol. 188, no. 1, pp. 123-128, 2007.

[30] W. M. Abd-Elhameed, E. H. Doha, and Y. H. Youssri, "New spectral second kind Chebyshev wavelets algorithm for solving linear and nonlinear second-order differential equations involving singular and Bratu type equations," Abstract and Applied Analysis, vol. 2013, Article ID 715756, 9 pages, 2013.

[31] R. Barrett, M. Berry, T. F. Chan et al., Templates for the Solution of Linear Systems: Building Blocks for Iterative Methods, Society for Industrial and Applied Mathematics (SIAM), 1994.

[32] R. S. Dembo, S. C. Eisenstat, and T. Steihaug, "Inexact Newton methods," SIAM Journal on Numerical Analysis, vol. 19, no. 2, pp. 400-408, 1982.

[33] Y.-C. Li, "A note on an identity of the gamma function and Stirling's formula," Real Analysis Exchange, vol. 32, no. 1, pp. 267-272, 2007.

[34] N. A. Khan, A. Ara, and N. A. Khan, "Fractional-order Riccati differential equation: analytical approximation and numerical results," Advances in Difference Equations, vol. 2013, article 185, 2013.

[35] Z. Odibat and S. Momani, "Modified homotopy perturbation method: application to quadratic Riccati differential equation of fractional order," Chaos, Solitons \& Fractals, vol. 36, no. 1, pp. 167-174, 2008.

[36] F. Yin, J. Song, J. H. Leng, and F. Lu, "Couple of the variational iteration method and fractional-order legendre functions method for fractional differential equations," The Scientific World Journal, vol. 2014, Article ID 928765, 9 pages, 2014.

[37] Y. Wang and Q. Fan, "The second kind Chebyshev wavelet method for solving fractional differential equations," Applied Mathematics and Computation, vol. 218, no. 17, pp. 8592-8601, 2012.

[38] W. A. Light, "Are Chebysheve expansions really better," Bulletin of the Institute of Mathematics and Its Applications, vol. 22, pp. 180-181, 1986. 


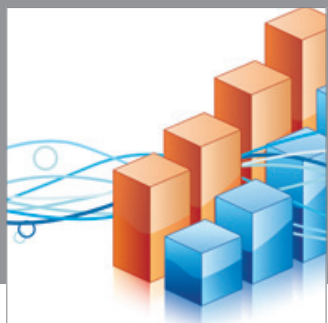

Advances in

Operations Research

mansans

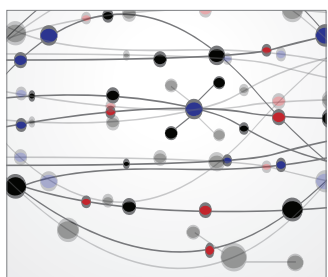

The Scientific World Journal
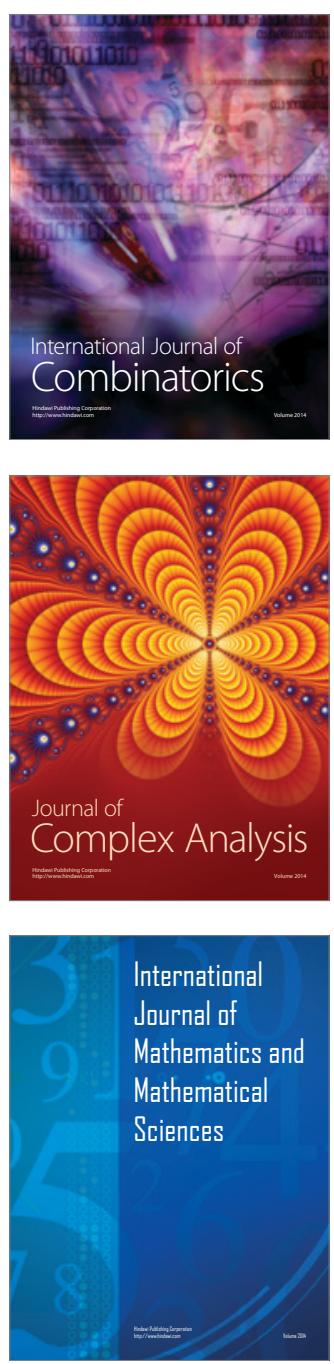
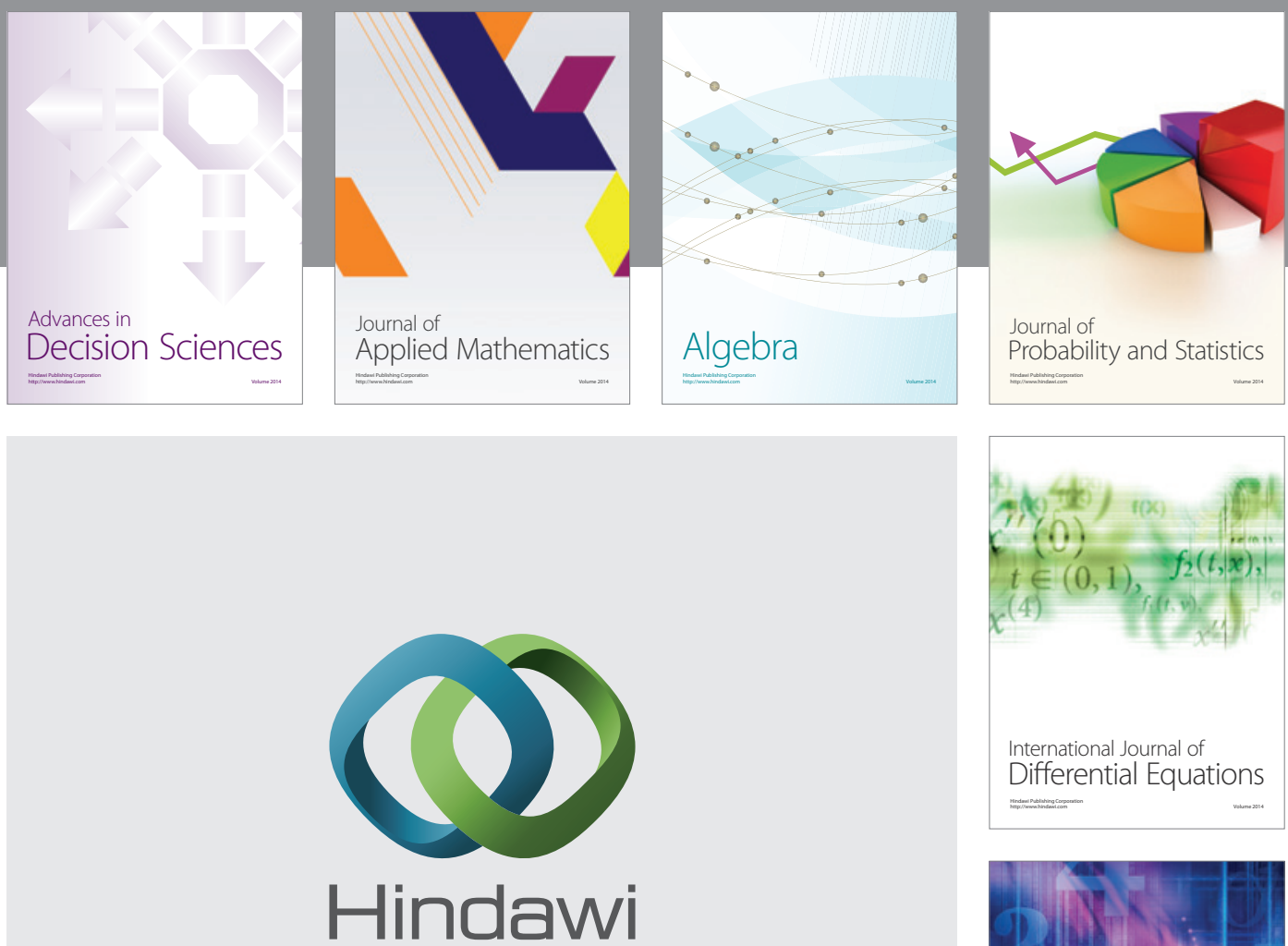

Submit your manuscripts at http://www.hindawi.com
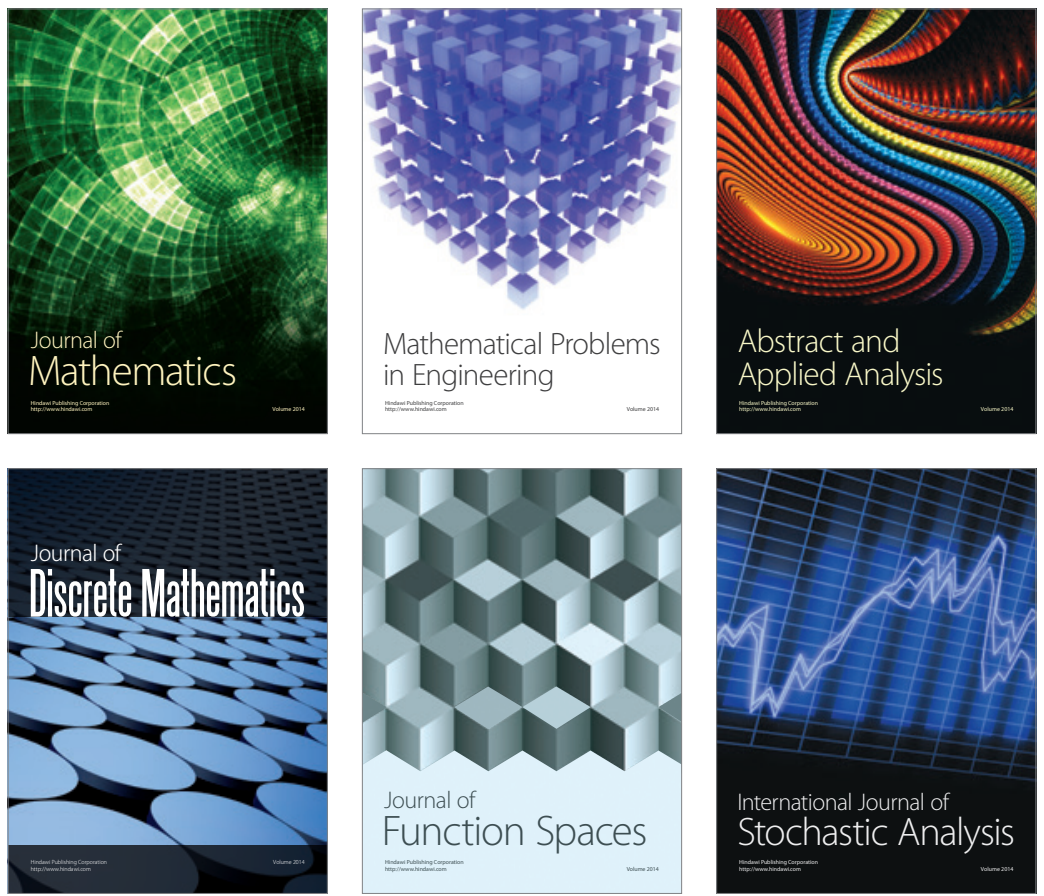

Journal of

Function Spaces

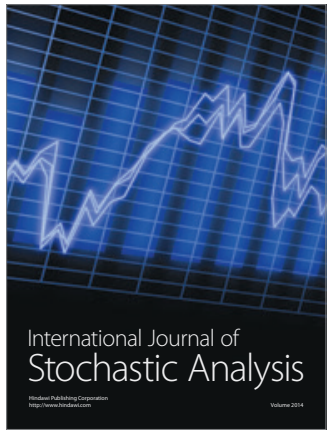

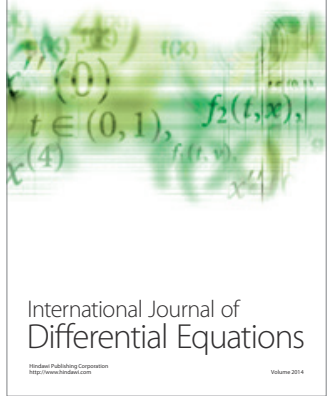
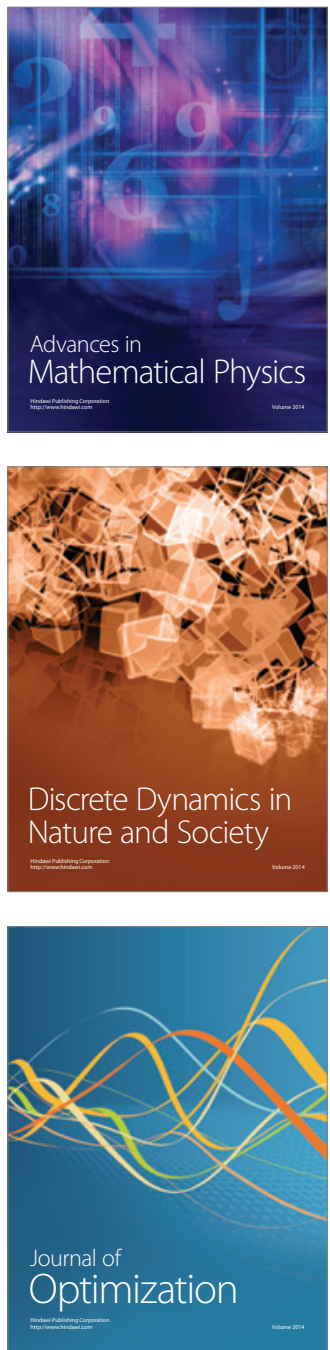